ISSN: 0210-1696

DOI: http://dx.doi.org/10.14201/scero20195015373

\title{
VALIDEZ DIAGNÓSTICA DE LA ESCALA DE DESARROLLO MERRILL PALMER-R UTILIZADA EN LA EVALUACIÓN DE ACCESO A LOS CENTROS DE DESARROLLO INFANTIL Y ATENCIÓN TEMPRANA
}

\section{Diagnostic validity of the Merrill Palmer- $R$ Development Scale used in the evaluation of access to Child Development Centers and Early Care}

\author{
Francisco Alcantud-Marín \\ Universitat de València. Facultad de Psicología. Departamento de Psicología Evolutiva y de la \\ Educación. Avd. Blasco Ibáñez, 21. 46010 Valencia \\ Francisco.Alcantud@uv.es \\ Javier PÉrez-Bou \\ Universidad Católica de Valencia San Vicente Mártir \\ Yurena Alonso-Esteban \\ Universidad Internacional de Valencia (Valencian International University)
}

Recepción: 27 de noviembre de 2017

Aceptación definitiva: 27 de junio de 2018

Resumen: La Escala de Desarrollo Merrill Palmer-R es de uso común en los Centros de Desarrollo Infantil y Atención Temprana. Suele utilizarse, al igual que otras escalas, en el proceso de evaluación inicial en el momento de alta en el centro y/o a lo largo de toda la intervención. Se presentan los resultados obtenidos en la aplicación de la escala en el Centro Universitario de Diagnóstico y Atención Temprana de la Universidad de Valencia, en el periodo comprendido entre 2011 y 2016. En total se han analizado 223 casos de niños y niñas menores de 42 meses, la mayor parte de ellos sin diagnóstico 
o con un diagnóstico provisional en el momento del alta. El objetivo de este artículo es determinar el valor diagnóstico de la escala Merrill Palmer-R y si el uso de la misma, en el momento del alta en el centro, facilita la elaboración de programas de intervención y la asignación de los recursos necesarios para su desarrollo. Para ello, se ha estudiado la clasificación de los casos en función de las puntuaciones en la MP-R mediante análisis de Clúster K-Means, obteniéndose cuatro grupos. Esta clasificación ha sido validada mediante análisis discriminante. La bondad de clasificación estadística alcanza el 93,7\%. Los cuatro grupos corresponden a cuatro perfiles diagnósticos según, fundamentalmente, la gravedad del caso y las áreas del desarrollo afectadas. Los grupos obtenidos se han contrastado con el diagnóstico real de cada caso. Se concluye que la escala es de utilidad en el proceso de evaluación inicial en el centro y se aconseja, siguiendo las recomendaciones del APA, añadir al diagnóstico final en el momento de baja en el centro un nivel de gravedad para ajustar las necesidades futuras de recursos.

Palabras clave: atención temprana; escalas de desarrollo, diagnóstico y cribado; Merrill Palmer-R.

Aвstracts: The Merrill Palmer-R Development Scale is commonly used in Child Development and Early Care Centers. It is usually used, like other scales, in the initial evaluation process at the time of discharge in the center and / or throughout the entire intervention. The results obtained in the application of the scale are presented in the University Center of Diagnostic and Early Care of the University of Valencia, in the period between 2011 and 2016. In total, 223 cases of children under 42 months have been analyzed. Most of them, or without diagnosis or with a provisional diagnosis at the time of discharge. The objective of this article is to determine the diagnostic value of the Merrill Palmer-R scale and if the use of it, at the time of discharge in the center, facilitates the development of intervention programs and the allocation of resources necessary for its development. For this, the classification of the cases according to the scores in the MP-R has been studied by means of Cluster K-Means analysis, obtaining four groups. This classification has been validated by discriminant analysis. The goodness of statistical classification reaches $93.7 \%$. The four groups correspond to four diagnostic profiles according to the severity of the case and the affected areas of development. The groups obtained have been contrasted with the real diagnosis of each case. It is concluded that the scale is useful in the initial evaluation process at the center and it is advised, following the recommendations of the APA, to add to the final diagnosis at the time of leaving the center, a level of severity to adjust the future needs of resources.

KEY WORDs: early attention; development scales; diagnosis and screening; Merrill Palmer-R.

\section{Introducción}

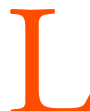

a Atención Temprana es el conjunto de acciones que se realiza sobre los niños con trastornos del desarrollo o riesgo de padecerlo y sus familias (GAT, 2000). Existen numerosas evidencias, y desde hace ya algún tiempo, sobre la eficacia de los programas de Atención Temprana (Casto y Mastropieri, 1986;

(C) Ediciones Universidad de Salamanca / CC BY-NC-ND

Siglo Cero, vol. 50 (1), n. ${ }^{\circ}$ 269, 2019, enero-marzo, pp. 53-73 
VALIDEZ DIAGNÓSTICA DE LA ESCALA DE DESARROLLO MERRILL PALMER-R UTILIZADA EN LA

EVALUACIÓN DE ACCESO A LOS CENTROS DE DESARROLLO INFANTIL Y ATENCIÓN TEMPRANA FRANCISCO ALCANTUD-MARÍN, JAVIER PÉREZ-BOU Y YURENA ALONSO-ESTEBAN

Shonkoff y Hauser-Cram, 1987; Guralnick y Bennett, 1987; Guralnick, 1998; Barnett, 1995; Reynolds, Temple, Robertson y Mann, 2001; McCormick, Brooks-Gum, Buka, Goldman y Yu, 2006, entre otras).

En la mayor parte de las Comunidades Autónomas de España, la Atención Temprana con independencia del modelo de intervención por el que se opte, las actividades se desarrollan en los Centros de Desarrollo Infantil y Atención Temprana (en adelante CDIAT) (GAT, 2011). La prestación de un servicio de estas características implica el trabajo de un equipo multidisciplinar que debe tomar decisiones sobre qué hacer y cuándo hacerlo, en colaboración con profesionales de los servicios de sanidad y educación de forma transversal (King et al., 2009). Los CDIAT son centros, salvo excepciones, públicos o subvencionados por recursos públicos que deben ser administrados bajo el principio de eficacia, economía y eficiencia. En este sentido, estamos obligados a ser lo más productivos posible al menor coste. Ello implica que debemos atender al máximo de usuarios, con el máximo rigor y calidad. Por otra parte, los CDIAT son centros generalistas en los que se atienden todo tipo de trastornos. Esta es una regla más o menos general en todo el Estado, aunque existen excepciones como es el caso de los trastornos visuales atendidos por la ONCE, o en algunas comunidades autónomas en las que existen centros específicos para trastornos concretos, al margen de las iniciativas privadas que pueden existir en todo el Estado. En ocasiones incluso siendo las entidades que organizan los servicios de los CDIAT, asociaciones de familias de niños/as afectados por determinado trastorno (síndrome de Down, síndrome de Prader-Willi, Parálisis Cerebral Infantil, entre otras), por la evolución de la prevalencia de los diferentes trastornos, se atiende de forma general a todo tipo de trastornos del desarrollo. A estos los denominaremos centros generalistas frente a los centros especializados que solo atienden un tipo de patología o patologías afines.

La derivación a los CDIAT se puede producir con cierto nivel de incertidumbre, en unos casos solo se ha observado un retraso o trastorno en el desarrollo, en otros pertenece a una población de riesgo con manifestación o no de síntomas y, por último, los casos con diagnóstico. Aun teniendo un diagnóstico claro, la asignación de recursos en el CDIAT se realiza en función de la gravedad del trastorno o el área o áreas del desarrollo afectadas. Se hace necesaria una evaluación inicial. Este proceso se inicia con una primera entrevista donde se recoge información de diferentes fuentes (familia, informes médicos, informes educativos, informes sociales, pruebas objetivas, observación directa, etc.). El objetivo es conocer la realidad del niño/a y su contexto familiar para determinar la necesidad de desarrollar un programa de intervención y evaluar posteriormente sus resultados. En definitiva, en este proceso se suelen diferenciar varias fases:

a) Recogida de información en la entrevista inicial con la familia:

a. Entrevista personal.

b. Documentación aportada por la familia: informes médicos, informes psicológicos, informes sociales, etc.

b) Primera impresión diagnóstica: sobre esta primera impresión se inicia el proceso de observación y evaluación del niño/a con la finalidad de confirmarla o refutarla. Se determinan las pruebas a realizar que serán: 
VALIDEZ DIAGNÓSTICA DE LA ESCALA DE DESARROLLO MERRILL PALMER-R UTILIZADA EN LA

EVALUACIÓN DE ACCESO A LOS CENTROS DE DESARROLLO INFANTIL Y ATENCIÓN TEMPRANA FRANCISCO ALCANTUD-MARÍN, JAVIER PÉREZ-BOU Y YURENA ALONSO-ESTEBAN

a. Pruebas Objetivas Estandarizadas.

b. Registros observacionales (videos del niño en contexto natural, videos domésticos aportados por la familia, videos de las sesiones de evaluación y videos de las sesiones iniciales).

c) Elaboración del informe de devolución a las familias y primera propuesta de PIT (Programa de Intervención Terapéutica) si procede o, en su caso, programación de seguimiento del niño/a.

Este proceso de evaluación inicial debe ser rápido para poder: a) dar una respuesta a la familia aminorando en lo posible la tensión producida por la incertidumbre y b) iniciar un programa de intervención individualizado lo más pronto posible. Durante esta fase de evaluación, en la mayoría de los CDIAT se utilizan diferentes instrumentos de medida [Brunet-Lézine Revisada (Josse y Pereda, 1997); Escalas Bayley III (Bayley, 2006); Inventario Battelle (Newborg, 2005); Escala Merrill Palmer-R (Roid y Sampers, 2004), entre otras muchas].

En este artículo se presentan los resultados del uso de la Escala de Desarrollo Merrill Palmer Revisada (Roid y Sampers, 2004) (en adelante MP-R) como herramienta de evaluación en el proceso de ingreso en un CDIAT. Este estudio se inició con motivo de la puesta en marcha del sistema de gestión del CUDAP ${ }^{1}$ (Pérez, 2015). El objetivo en aquel momento fue encontrar criterios que ayudaran a orientar las decisiones sobre la asignación de recursos y la elaboración del propio Programa de Intervención Terapéutica. En este artículo se ha actualizado la base de datos para su publicación.

\section{Escalas de Desarrollo Merrill Palmer-R}

La MP-R fue publicada por primera vez en 1931 (Stutsman, 1931) y alcanzó un alto prestigio internacional. En 2004 se publicó la versión revisada (Roid y Sampers, 2004) y en el 2011 la primera versión revisada española. La Escala de Desarrollo MP-R se compone de varias subpruebas con la estructura siguiente:

a) Batería cognitiva:
a. Índice Global (IG).
b. Cognición (C).
c. Motricidad Fina (MF).
d. Lenguaje Receptivo (LR) o Lenguaje Infantil (LI) para niños/as menores del año.

Escalas complementarias:
e. Memoria (M) o Memoria Infantil (MI) para niños/as menores del año.
f. Velocidad de Procesamiento (V).
g. Coordinación Visomotora (CV).

1 CUDAP: Centre Universitari de Diagnostic i Atenció Primerença de la Universitat de Valencia. 
VALIDEZ DIAGNÓSTICA DE LA ESCALA DE DESARROLLO MERRILL PALMER-R UTILIZADA EN LA

EVALUACIÓN DE ACCESO A LOS CENTROS DE DESARROLLO INFANTIL Y ATENCIÓN TEMPRANA FRANCISCO ALCANTUD-MARÍN, JAVIER PÉREZ-BOU Y YURENA ALONSO-ESTEBAN

b) Escala Motricidad Gruesa (MG).

c) Escalas complementarias y de observación.

a. Escala Lenguaje Expresivo-Examinador (LE-E).

b. Escala Lenguaje Expresivo-Padres (LE-P).

i. Lenguaje Expresivo-padres (LE-P).

ii. Lenguaje Expresivo infantil (LEI) para niños/as menores del año.

c. Escala Socioemocial (SE).

d. Escala Estilo de Temperamento.

e. Escala Conducta Adaptativa y Autocuidado (CAA).

Hasta los 12 meses se aplican las formas infantiles (Lenguaje Infantil, Memoria Infantil y Lenguaje Expresivo Infantil) y a partir de esta edad y hasta la edad techo 78 meses (6 años y medio) se aplican las escalas estándar. La MP-R fue diseñada como herramienta de identificación de trastornos del desarrollo en edades tempranas y específicamente para ser usada en medidas repetidas en el seguimiento del progreso y evolución de las habilidades en las áreas recogidas en el IDEA². La MP-R aportaba como novedad, en su momento, las puntuaciones normalizadas de desarrollo basadas en el modelo de la Teoría de Respuesta del Ítem que permitían analizar y realizar el seguimiento de la evolución del desarrollo del niño. Este mismo modelo de puntuación se ha utilizado en las nuevas versiones de las escalas Leiter-R (Roid y Miller, 1997), Stanford-Binet 5 (Roid, 2003) y Bayley III (Bayley, 2006), entre otras. En nuestro entorno, la escala del desarrollo MP-R ha manifestado su valor predictivo al menos durante el primer año de vida (Alcantud y Alonso, 2016).

\section{Valor diagnóstico de la MP-R}

En el manual de la escala MP-R (Roid y Sampers, 2004) se incluye un estudio diferencial en base a las puntuaciones típicas obtenidas en la administración de la escala, determinándose perfiles diferenciales según diagnóstico. En un estudio posterior (Peters, 2013), se realiza una comparación entre estos perfiles y los de dos grupos de niños/as, uno con desarrollo promedio (neurotípico) y otro grupo de niños/as con diagnóstico de Trastorno del Espectro Autista. Nosotros hemos recopilado los datos de ambos estudios en la Tabla 1.

Los perfiles entre los diferentes grupos de diagnóstico varían tanto en la puntuación alcanzada como en los máximos y mínimos que manifiestan cada uno de ellos y sus correspondientes dispersiones (desviaciones típicas). Es de destacar que el perfil de niños/as de desarrollo neurotípico mantiene una línea más o menos recta a través de todas las áreas de evaluación, siendo la media próxima a la media poblacional estimada 100 y la desviación típica próxima a 15. En cambio, en los perfiles del resto de grupos se observan diferencias importantes entre las diferentes áreas. De estos datos se desprende que podría ser posible determinar perfiles diferenciales según las

2 IDEA: Individuals with Disabilities Education Act 2004-2006. 
VALIDEZ DIAGNÓSTICA DE LA ESCALA DE DESARROLLO MERRILL PALMER-R UTILIZADA EN LA EVALUACIÓN DE ACCESO A LOS CENTROS DE DESARROLLO INFANTIL Y ATENCIÓN TEMPRANA FRANCISCO ALCANTUD-MARÍN, JAVIER PÉREZ-BOU Y YURENA ALONSO-ESTEBAN

puntuaciones en esta escala que, o bien ayuden en la orientación del desarrollo del programa de intervención, o bien nos den información sobre el diagnóstico posible y la gravedad del trastorno.

\begin{tabular}{|c|c|c|c|c|c|c|c|c|c|c|c|c|c|c|c|c|}
\hline \multicolumn{17}{|c|}{$\begin{array}{c}\text { TABLA 1. Medias y desviaciones de las diferentes escalas de la MP-R según grupo } \\
\text { diagnóstico }\end{array}$} \\
\hline & \multicolumn{12}{|c|}{ Resultados de Roid y Sampers, 2004} & \multicolumn{4}{|c|}{$\begin{array}{c}\text { Resultados } \\
\text { de Peters, } 2013\end{array}$} \\
\hline & \multicolumn{2}{|c|}{$\begin{array}{l}\text { Retraso } \\
\text { cognitivo }\end{array}$} & \multicolumn{2}{|c|}{$\begin{array}{c}\text { Niños/as } \\
\text { prematuros }\end{array}$} & \multicolumn{2}{|c|}{$\begin{array}{c}\text { Trastornos } \\
\text { del habla y } \\
\text { lenguaje }\end{array}$} & \multicolumn{2}{|c|}{ Sordo } & \multicolumn{2}{|c|}{$\begin{array}{l}\text { Retraso } \\
\text { motor }\end{array}$} & \multicolumn{2}{|c|}{ TEA } & \multicolumn{2}{|c|}{ TEA } & \multicolumn{2}{|c|}{$\begin{array}{l}\text { Desarrollo } \\
\text { neurotípico }\end{array}$} \\
\hline & $\overline{\mathrm{x}}$ & $\sigma$ & $\overline{\mathrm{x}}$ & $\sigma$ & $\overline{\mathrm{x}}$ & $\sigma$ & $\overline{\mathrm{x}}$ & $\sigma$ & $\overline{\mathrm{x}}$ & $\sigma$ & $\overline{\mathrm{x}}$ & $\sigma$ & $\overline{\mathrm{x}}$ & $\sigma$ & $\bar{x}$ & $\sigma$ \\
\hline Cognitiva & 49,6 & 26,4 & 94,2 & 22,3 & 85,8 & 22,7 & 92,5 & 21,9 & 76,5 & 35,8 & 58,7 & 30,7 & 70,7 & 38,6 & 99,0 & 14,58 \\
\hline Motricidad fina & 47,0 & 26,5 & 90,8 & 19,6 & 87,2 & 21,5 & 94,4 & 21,0 & 72,3 & 33,9 & 58,9 & 26,3 & 72,1 & 38,6 & 100,8 & 13,30 \\
\hline $\begin{array}{l}\text { Lenguaje } \\
\text { receptivo }\end{array}$ & & & & & 76,4 & 15,2 & 72,8 & 19,5 & 73,4 & 25,4 & 54,2 & 15,6 & 65,5 & 40,5 & 98,0 & 14,91 \\
\hline Memoria & 53,6 & 26,0 & & & 87,3 & 21,1 & \begin{tabular}{|l|l}
95,4 \\
\end{tabular} & 20,1 & 73,9 & 31,0 & 68,3 & 22,9 & 65,0 & 37,4 & 99,8 & 13,66 \\
\hline $\begin{array}{l}\text { Velocidad } \\
\text { procesamiento }\end{array}$ & 60,3 & 24,1 & & & 86,5 & 19,0 & 93,4 & 18,9 & 80,6 & 38,8 & 69,4 & 23,2 & 84,4 & 34,0 & 99,7 & 15,54 \\
\hline $\begin{array}{l}\text { Coordinación } \\
\text { visomotora }\end{array}$ & 48,9 & 22,7 & 92,1 & 18,0 & 88,1 & 20,1 & 95,5 & 21,6 & 74,3 & 33,3 & 57,1 & 23,2 & 62,6 & 33,9 & 100,4 & 12,73 \\
\hline Motricidad gruesa & 54,3 & 26,3 & 90,9 & 24,0 & 90,6 & 22,0 & 83,9 & 25,4 & 70,9 & 31,3 & 75,5 & 15,0 & 74,5 & 20,1 & 100,0 & 13,19 \\
\hline $\begin{array}{l}\text { Lenguaje } \\
\text { expresivo }\end{array}$ & & & & & & & & & & & & & 59,8 & 25,9 & 102,0 & 16,36 \\
\hline Socioemocional & & & & & & & & & & & & & 64,0 & 26,0 & 100,6 & 15,92 \\
\hline $\begin{array}{l}\text { Conducta } \\
\text { adaptativa }\end{array}$ & & & & & & & & & & & & & 74,1 & 22,8 & 97,2 & 12,60 \\
\hline $\mathrm{N}$ & \multicolumn{2}{|c|}{20} & \multicolumn{2}{|c|}{36} & \multicolumn{2}{|c|}{42} & \multicolumn{2}{|c|}{16} & \multicolumn{2}{|c|}{15} & \multicolumn{2}{|c|}{17} & 30 & & \multicolumn{2}{|c|}{$\partial 0$} \\
\hline Edad media & \multicolumn{2}{|c|}{56} & \multicolumn{2}{|c|}{11} & \multicolumn{2}{|c|}{47} & \multicolumn{2}{|c|}{49} & \multicolumn{2}{|r|}{$J$} & \multicolumn{2}{|c|}{53} & \multicolumn{4}{|c|}{36 a 72 meses } \\
\hline
\end{tabular}

\section{Método}

El objetivo de este estudio es conocer el valor pronóstico de la MP-R en el momento de alta en el CDIAT respecto al diagnóstico alcanzado en el momento de baja en el centro, con la finalidad de poder utilizar esta información a la hora de asignar recursos del centro.

\section{Muestra}

Los datos utilizados para el análisis se obtendrán de un único CDIAT. El CUDAP se creó en el 2006 por acuerdo entre la Conselleria de Bienestar Social de la Generalitat 
VALIDEZ DIAGNÓSTICA DE LA ESCALA DE DESARROLLO MERRILL PALMER-R UTILIZADA EN LA EVALUACIÓN DE ACCESO A LOS CENTROS DE DESARROLLO INFANTIL Y ATENCIÓN TEMPRANA FRANCISCO ALCANTUD-MARÍN, JAVIER PÉREZ-BOU Y YURENA ALONSO-ESTEBAN

Valenciana y la Universitat de València, empezando el servicio en octubre del 2008. Hasta diciembre del 2016 ha atendido un total de 800 casos. Como criterios de selección de la muestra para este estudio se han determinado los siguientes:

a) Los casos seleccionados deben disponer de los datos completos de la aplicación de la escala MP-R. En este sentido, como la versión española de la escala se publica en mayo del 2011, no se consideran los casos dados de baja con anterioridad a esta fecha. Tampoco se consideran los casos que por algún motivo no tengan los datos completos de la MP-R con posterioridad a la misma.

b) Se excluyen también los expedientes que con fecha 31 de diciembre del 2016 se mantienen activos en el centro.

c) Se excluyen los/as niños/as de más de 42 meses de edad.

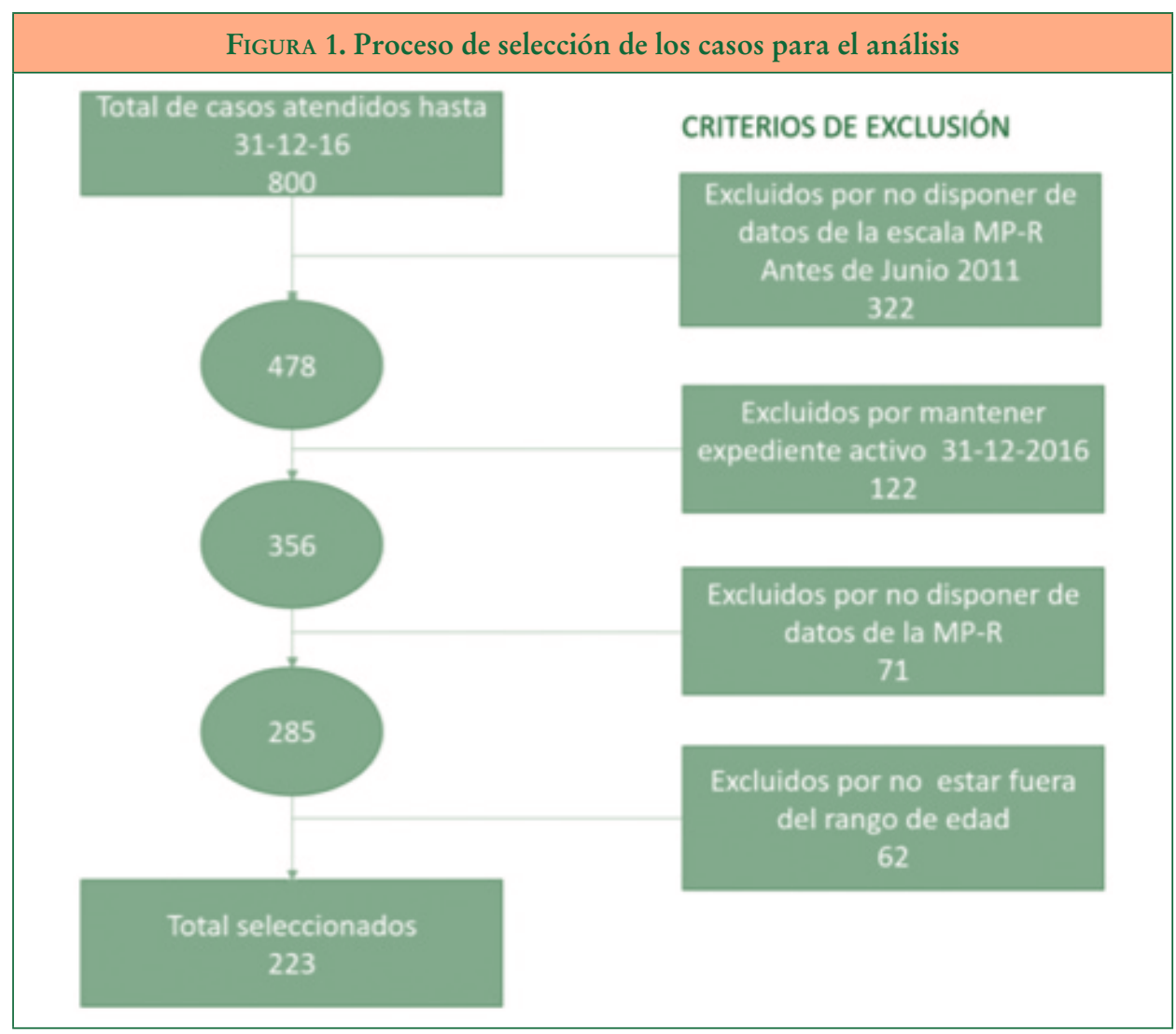

En definitiva, el grupo de niños/as objeto del estudio se compone de 223 niños/as menores 42 meses que fueron evaluados y atendidos en el CUDAP en el periodo de tiempo establecido (2011-2016). En la Tabla 2, se presenta la distribución de los casos 
VALIDEZ DIAGNÓSTICA DE LA ESCALA DE DESARROLLO MERRILL PALMER-R UTILIZADA EN LA

EVALUACIÓN DE ACCESO A LOS CENTROS DE DESARROLLO INFANTIL Y ATENCIÓN TEMPRANA FRANCISCO ALCANTUD-MARÍN, JAVIER PÉREZ-BOU Y YURENA ALONSO-ESTEBAN

seleccionados según la edad, género y motivo de alta en el centro, siguiendo la ODAT ${ }^{3}$ (GAT, 2004). Destacamos que el motivo más importante de alta en el centro es el de "riesgo biológico", siendo la "prematuridad" el riesgo biológico más frecuente.

Tabla 2. Distribución del número de casos seleccionados, desde 2011 hasta 2016 según edad y causa de alta en el centro

\begin{tabular}{|c|c|c|c|c|c|c|c|c|c|c|c|c|c|c|c|}
\hline & \multicolumn{2}{|c|}{$\begin{array}{c}\text { hasta } \\
6 \text { meses }\end{array}$} & \multicolumn{2}{|c|}{$\begin{array}{l}\text { de } 7 \\
\text { a } 12 \\
\text { meses }\end{array}$} & \multicolumn{2}{|c|}{$\begin{array}{l}\text { de } 13 \\
\text { a } 18 \\
\text { meses }\end{array}$} & \multicolumn{2}{|c|}{$\begin{array}{c}\text { de } 19 \\
\text { a } 24 \\
\text { meses }\end{array}$} & \multicolumn{2}{|c|}{$\begin{array}{c}\text { de } 25 \\
\text { a } 30 \\
\text { meses }\end{array}$} & \multicolumn{2}{|c|}{$\begin{array}{c}\text { de } 31 \\
\text { a } 36 \\
\text { meses }\end{array}$} & \multicolumn{2}{|c|}{\begin{tabular}{|c|}
37 \\
meses \\
a 42 \\
meses \\
\end{tabular}} & \multirow[t]{2}{*}{ Total } \\
\hline & $\mathrm{V}$ & $\mathrm{M}$ & $\mathrm{V}$ & $\mathrm{M}$ & $\mathrm{V}$ & $\mathrm{M}$ & $\mathrm{V}$ & $\mathrm{M}$ & $\mathrm{V}$ & $\mathrm{M}$ & $\mathrm{V}$ & $\mathrm{M}$ & $\mathrm{V}$ & $\mathrm{M}$ & \\
\hline Riesgo biológico & 12 & 13 & 19 & 7 & 6 & 2 & 9 & 4 & 13 & 10 & 9 & 3 & 12 & 5 & 124 \\
\hline Síndrome genético o biológico & 0 & 0 & 1 & 1 & 0 & 1 & 1 & 1 & 0 & 1 & 1 & 0 & 3 & 0 & 10 \\
\hline Prematuridad extrema (menos de $28 \mathrm{~s} / \mathrm{g}$ ) & 1 & 3 & 4 & 2 & 0 & 0 & 0 & 0 & 0 & 1 & 0 & 0 & 0 & 1 & 12 \\
\hline Prematuridad 29 a $34 \mathrm{~s} / \mathrm{g}$ & 3 & 5 & 9 & 1 & 3 & 0 & 0 & 0 & 0 & 3 & 0 & 0 & 0 & 2 & 26 \\
\hline Prematuridad tardía 35 a $38 \mathrm{~s} / \mathrm{g}$ & 5 & 1 & 2 & 0 & 1 & 1 & 3 & 2 & 7 & 3 & 6 & 3 & 4 & 1 & 39 \\
\hline Complicaciones en el parto & 0 & 1 & 1 & 2 & 1 & 0 & 3 & 0 & 3 & 1 & 1 & 0 & 3 & 0 & 16 \\
\hline Reproducción asistida & 0 & 1 & 0 & 0 & 0 & 0 & 0 & 1 & 3 & 0 & 1 & 0 & 2 & 0 & 8 \\
\hline Problemas neurológicos & 3 & 2 & 2 & 1 & 1 & 0 & 2 & 0 & 0 & 1 & 0 & 0 & 0 & 1 & 13 \\
\hline Retraso psicomotor & 1 & 1 & 2 & 1 & 5 & 6 & 2 & 2 & 3 & 1 & 2 & 0 & 6 & 1 & 33 \\
\hline Retraso en el lenguaje & 0 & 0 & 0 & 0 & 2 & 0 & 4 & 2 & 11 & 5 & 6 & 2 & 14 & 2 & 48 \\
\hline Retraso comunicación/relación & 0 & 0 & 0 & 0 & 1 & 0 & 2 & 2 & 6 & 1 & 3 & 0 & 3 & 1 & 19 \\
\hline Total por género & 13 & 14 & 21 & 8 & 14 & 8 & 17 & 10 & 32 & 17 & 20 & 5 & 35 & 9 & \\
\hline Total por edad & 27 & & 2 & & 22 & & 2 & & 44 & & 2. & & 4 & 4 & 223 \\
\hline
\end{tabular}

Dado que todos los casos incluidos en este estudio habían sido dados de baja en el CUDAP (expedientes no activos), se analizaron los expedientes. Es importante destacar aquí que en esta revisión los niños/as que se dieron de alta en el centro con más de 25 meses, aunque el motivo expuesto por los padres fuera "retraso en el lenguaje", "retraso psicomotor" u otro, al analizar la anamnesis de caso se observó que en algunos casos existía información sobre el parto y la gestación y cuando así lo justificara, se reclasificaron los motivos de alta por el correspondiente "Riesgo Biológico".

$\mathrm{Al}$ analizar los expedientes se asignó el diagnóstico según el DSM-5 (APA, 2013) en el momento de la baja en el centro. La distribución de casos se presenta en la Tabla 3. Obsérvese como, dada la edad de los/as niños/as en el momento de baja en el centro, existe un porcentaje alto de casos etiquetados como "Trastorno Global del Desarrollo”. Tal como indica el manual del DSM-5 (p. 23), este diagnóstico se reserva a menores de 5 años cuando la gravedad y sintomatología no se puede valorar de forma fiable por la edad y se hace necesaria una evaluación posterior. En definitiva, se ha utilizado como "cajón de sastre" cuando no existen evidencias de un diagnóstico

3 ODAT: Organización Diagnóstica en Atención Temprana.

(C) Ediciones Universidad de Salamanca / CC BY-NC-ND

Siglo Cero, vol. 50 (1), n. ${ }^{\circ}$ 269, 2019, enero-marzo, pp. 53-73 
VALIDEZ DIAGNÓSTICA DE LA ESCALA DE DESARROLLO MERRILL PALMER-R UTILIZADA EN LA EVALUACIÓN DE ACCESO A LOS CENTROS DE DESARROLLO INFANTIL Y ATENCIÓN TEMPRANA FRANCISCO ALCANTUD-MARÍN, JAVIER PÉREZ-BOU Y YURENA ALONSO-ESTEBAN

concreto, aunque sí es evidente un trastorno del desarrollo. Obsérvese que existe un porcentaje importante $(28,70 \%)$ de casos que son dados de baja en el centro sin signos significativos de trastornos o retrasos en el desarrollo (alcanzando la normalidad en el momento de baja en el centro). Es decir, accedieron al CDIAT por alguno de los motivos de riesgo y tras la evaluación o al alcanzar la edad reglamentaria se les dio de baja por no presentar síntomas de retraso o trastorno del desarrollo.

\begin{tabular}{|c|c|c|c|c|c|c|c|c|c|c|c|c|c|}
\hline & \multicolumn{2}{|c|}{$\begin{array}{c}\text { Normalidad } \\
\text { (sin } \\
\text { diagnóstico) }\end{array}$} & \multicolumn{2}{|c|}{$\begin{array}{l}\text { Trastorno } \\
\text { Global del } \\
\text { Desarrollo }\end{array}$} & \multicolumn{2}{|c|}{$\begin{array}{c}\text { Discapacidad } \\
\text { Intelectual }\end{array}$} & \multicolumn{2}{|c|}{$\begin{array}{c}\text { Trastorno } \\
\text { del Espectro } \\
\text { Autista }\end{array}$} & \multicolumn{2}{|c|}{$\begin{array}{l}\text { Trastorno } \\
\text { Motor }\end{array}$} & \multicolumn{2}{|c|}{$\begin{array}{c}\text { Trastorno del } \\
\text { Lenguaje }\end{array}$} & \multirow[t]{2}{*}{ Total } \\
\hline & V & $\mathrm{M}$ & $\mathrm{V}$ & $\mathrm{M}$ & $\mathrm{V}$ & $\mathrm{M}$ & $\mathrm{V}$ & $\mathrm{M}$ & $\mathrm{V}$ & $\mathrm{M}$ & $\mathrm{V}$ & $\mathrm{M}$ & \\
\hline Riesgo biológico: & 25 & 18 & 22 & 9 & 5 & 3 & 24 & 6 & 0 & 5 & 4 & 1 & 122 \\
\hline Síndrome genético o biológico & 0 & 0 & 3 & 0 & 3 & 3 & 0 & 0 & 0 & 1 & 0 & 0 & 10 \\
\hline Prematuridad extrema (menos de $28 \mathrm{~s}$ ) & 4 & 3 & 1 & 3 & 0 & 0 & 0 & 0 & 0 & 1 & 0 & 0 & 12 \\
\hline Prematuridad 29 a $34 \mathrm{~s} / \mathrm{g}$ & 4 & 5 & 8 & 2 & 2 & 0 & 3 & 0 & 0 & 0 & 1 & 1 & 26 \\
\hline Prematuridad tardía 35 a 38 s/g & 13 & 4 & 4 & 2 & 0 & 0 & 9 & 4 & 0 & 1 & 2 & 0 & 39 \\
\hline Complicaciones en el parto & 0 & 3 & 2 & 1 & 0 & 0 & 10 & 0 & 0 & 0 & 0 & 0 & 16 \\
\hline Reproducción asistida & 2 & 1 & 1 & 0 & 0 & 0 & 1 & 1 & 0 & 0 & 0 & 0 & 6 \\
\hline Problemas neurológicos & 2 & 2 & 3 & 1 & 0 & 0 & 1 & 1 & 0 & 2 & 1 & 0 & 13 \\
\hline Retraso psicomotor & 6 & 1 & 11 & 8 & 2 & 0 & 0 & 0 & 2 & 3 & 0 & 0 & 33 \\
\hline Retraso en el lenguaje & 8 & 3 & 6 & 1 & 0 & 0 & 2 & 1 & 0 & 0 & 21 & 6 & 48 \\
\hline Retraso comunicación/relación & 3 & 0 & 5 & 4 & 0 & 0 & 3 & 0 & 0 & 0 & 4 & 0 & 19 \\
\hline Total por género & 42 & 22 & 44 & 22 & 7 & 3 & 29 & 7 & 2 & 8 & 29 & 7 & \\
\hline Total por diagnóstico & \multicolumn{2}{|c|}{64} & \multicolumn{2}{|c|}{65} & \multicolumn{2}{|c|}{10} & \multicolumn{2}{|c|}{36} & \multicolumn{2}{|c|}{10} & \multicolumn{2}{|c|}{36} & 223 \\
\hline
\end{tabular}

\section{Análisis de datos}

En la Tabla 4 se presentan las puntuaciones medias y desviaciones típicas de las diferentes subpruebas de la MP-R para cada grupo de edad y para el total de los sujetos seleccionados. Para los análisis optamos por no considerar las puntuaciones generales como IG y ITL por tratarse de combinaciones lineales de las diferentes subpruebas ya incluidas y, por tanto, no aportar información discriminante nueva. Tampoco se incluyeron las variables de memoria y velocidad de procesamiento debido a que no se aplicaba en niños/as menores de 18 meses y formar parte en definitiva de la subprueba de cognición. Por tanto, las variables que se consideraron para el análisis fueron: Cognición, Motricidad Fina, Motricidad Gruesa, Coordinación Visomotora, Lenguaje Receptivo, Lenguaje Expresivo, Desarrollo Socioemocional y Conducta adaptativa y autocuidado.

Los valores medios y desviaciones estándar, al tratarse de puntuaciones tipificadas $(\overline{\mathrm{X}}=100$ y $\sigma=15)$, deberían tender hacia esos valores cuando el tamaño del grupo 
VALIDEZ DIAGNÓSTICA DE LA ESCALA DE DESARROLLO MERRILL PALMER-R UTILIZADA EN LA

EVALUACIÓN DE ACCESO A LOS CENTROS DE DESARROLLO INFANTIL Y ATENCIÓN TEMPRANA FRANCISCO ALCANTUD-MARÍN, JAVIER PÉREZ-BOU Y YURENA ALONSO-ESTEBAN

tiende hacia infinito. No obstante, se observa que existen desviaciones significativas al tratarse de grupos de niños con trastornos del desarrollo.

TABLA 4. Medias y desviaciones típicas de las subpruebas de la escala MP-R

\begin{tabular}{|c|c|c|c|c|c|c|c|c|}
\hline & Cognición & $\begin{array}{c}\text { Motricidad } \\
\text { Fina }\end{array}$ & $\begin{array}{c}\text { Motricidad } \\
\text { Gruesa }\end{array}$ & $\begin{array}{c}\text { Coordinación } \\
\text { Visomotora } \\
\end{array}$ & $\begin{array}{l}\text { Lenguaje } \\
\text { Receptivo } \\
\end{array}$ & $\begin{array}{l}\text { Lenguaje } \\
\text { Expresivo } \\
\end{array}$ & $\begin{array}{c}\text { Socio- } \\
\text { emocional } \\
\end{array}$ & $\begin{array}{c}\text { Conducta } \\
\text { Adaptativa }\end{array}$ \\
\hline Normalidad & 98,31 & 98,58 & 96,63 & 96,87 & 92,72 & 78,67 & 89,53 & 95,54 \\
\hline$\sigma \quad 13,78$ & 11,46 & 14,25 & 12,05 & 26,47 & 29,07 & 12,02 & 13,05 & \\
\hline Trastorno Global & 76,55 & 74,27 & 79,54 & 69,32 & 73,36 & 62,21 & 81,34 & 85,63 \\
\hline $\begin{array}{lll}\text { del Desarrollo } & \sigma & 20,09 \\
\end{array}$ & 23,11 & 21,12 & 26,48 & 24,80 & 26,83 & 15,53 & 15,87 & \\
\hline Discapacidad & 68,70 & 68,10 & 72,20 & 60,70 & 65,13 & 60,75 & 76,30 & 71,40 \\
\hline$\sigma \quad 29,31$ & 35,75 & 28,52 & 40,83 & 31,03 & 22,77 & 18,00 & 18,12 & \\
\hline Trastorno del & 68,08 & 66,19 & 82,60 & 62,44 & 63,78 & 54,47 & 70,43 & 76,40 \\
\hline $\begin{array}{ll}\text { Espectro Autista } \sigma & 17,62 \\
\end{array}$ & 18,30 & 13,97 & 22,02 & 20,35 & 23,72 & 12,15 & 20,19 & \\
\hline Trastorno Motor & 93,50 & 92,60 & 65,80 & 89,70 & 99,75 & 87,89 & 92,11 & 91,89 \\
\hline$\sigma \quad 23,35$ & 18,51 & 28,62 & 19,21 & 14,75 & 20,18 & 10,27 & 10,40 & \\
\hline Trastorno del & 86,42 & 86,08 & 97,68 & 85,44 & 85,06 & 64,97 & 84,81 & 95,28 \\
\hline$\sigma \longdiv { 1 2 , 1 6 }$ & 13,88 & 10,96 & 15,52 & 16,38 & 18,14 & 13,77 & 12,71 & \\
\hline Total & 83,43 & 82,43 & 78,67 & 79,54 & 80,29 & 66,56 & 82,62 & 88,08 \\
\hline$\sigma \quad 20,86$ & 22,33 & 29,07 & 25,27 & 25,38 & 26,64 & 15,12 & 17,14 & \\
\hline \multicolumn{9}{|c|}{ ANOVA } \\
\hline $\mathrm{F}$ & 18,92 & 19,40 & 13,08 & 18,44 & 9,03 & 5,79 & 10,09 & 11,16 \\
\hline Significación & 0,00 & 0,00 & 0,00 & 0,00 & 0,00 & 0,00 & 0,00 & 0,00 \\
\hline \multicolumn{9}{|c|}{ Homogeneidad de varianza } \\
\hline Prueba Levene & 5,14 & 9,12 & 5,81 & 9,35 & 1,56 & 1,05 & 1,10 & 0,60 \\
\hline Significación & 0,00 & 0,00 & 0,00 & 0,00 & 0,17 & 0,39 & 0,36 & 0,70 \\
\hline
\end{tabular}

El análisis de las diferencias entre las medias, subprueba por subprueba y la homogeneidad de las varianzas nos demuestra que, aun teniendo razones $\mathrm{F}$ significativas en todos los casos entre los grupos formados por el diagnóstico, la falta de homogeneidad de la varianza hace que esta prueba en algunos casos carezca de consistencia. En definitiva, la variabilidad dentro de cada uno de los grupos por diagnóstico es muy elevada y el número de sujetos en cada grupo desigual por lo que difícilmente se podría extraer un perfil típico y característico.

Es interesante comparar los perfiles obtenidos en este estudio con los estudios precedentes. Al no utilizarse todas las subpruebas de la MP-R y los criterios diagnósticos de agrupación ser diferentes, nos obliga a tener que hacer dos comparaciones diferentes. Una entre los grupos TEA de los estudios de Roid y Sampers (2004), el estudio de Peters (2013) y los datos del estudio que aquí se presenta y otra con el resto de grupos posibles.

Si comparamos los perfiles del grupo de niños/as con desarrollo neurotípico del estudio de Sampers (o. c.) con los del presente estudio, se observa que ambos grupos 
VALIDEZ DIAGNÓSTICA DE LA ESCALA DE DESARROLLO MERRILL PALMER-R UTILIZADA EN LA

EVALUACIÓN DE ACCESO A LOS CENTROS DE DESARROLLO INFANTIL Y ATENCIÓN TEMPRANA FRANCISCO ALCANTUD-MARÍN, JAVIER PÉREZ-BOU Y YURENA ALONSO-ESTEBAN

tienen un comportamiento semejante con la excepción de la prueba de lenguaje expresivo. Este hecho podemos considerarlo como normal, dado que los niños/as de este estudio en algún momento presentaron un retraso o alteración en su desarrollo, aunque en el momento del baja en el centro se haya considerado como "no significativo" (la puntuación en esta subprueba es mayor a la $\bar{X} \pm 1 \sigma$ ). Por otra parte, si comparamos los tres perfiles de niños/as que han sido diagnosticados como Trastornos del Espectro Autista, observamos que el perfil de los niños del estudio de Sampers (o. c.) corre de forma paralela a los datos obtenidos en el CUDAP con ligeras diferencias. Lo mismo ocurre con los perfiles de los grupos diagnosticados como "Discapacidad Intelectual” y "Trastorno Generalizado del Desarrollo" que las diferencias radican en la magnitud de las puntuaciones aunque el perfil adquiere un recorrido similar.

Dadas estas evidencias, se intenta agrupar estadísticamente y de forma libre a los casos seleccionados en función de sus puntuaciones en la escala MP-R. Para ello se llevará a cabo la técnica de análisis de clúster. Esta técnica de estadística multivariante tiene como finalidad dividir un conjunto de objetos caracterizados por un número de variables métricas en grupos de forma que los perfiles de los objetos en un mismo grupo tengan la máxima homogeneidad (fuerte cohesión interna del grupo) y, simultáneamente, los grupos entre sí sean lo más diferentes posible (máxima variación entre grupos) (Escudero, 1977; Alcantud, 1985; Aldenderfer, 1985). Esta técnica ha sido muy utilizada en psicología a la hora de determinar grupos de individuos según sus características psicológicas. Belinchón y Olivar (2003) estudiaron los perfiles de funcionamiento psicológico de un grupo de 30 niños y adolescentes con diagnóstico de Autismo/TGD y otros trastornos psicopatológicos. En la misma línea, CrespoEguílaz y Narbona (2006) utilizan esta técnica para determinar la evidencia empírica de subtipos de los Trastornos Específicos del Lenguaje o las características del trastorno de aprendizaje procedimental (Crespo-Eguílaz y Narbona, 2009). No obstante, la eficacia de la clasificación depende tanto de la calidad y capacidad discriminadora de las variables utilizadas como del número de ellas, tamaño del grupo, dispersión o variabilidad de las puntuaciones, etc. (Alcantud, 1985).

Con la finalidad de validar los grupos obtenidos, se suelen utilizar criterios externos que, en nuestro caso, debería ser el diagnóstico de cada niño/a. No obstante, la diferencia en la gravedad de cada caso dentro de un mismo diagnóstico hace que la validez del criterio sea un punto crítico a tener en cuenta para valorar los resultados de la clasificación obtenida (Clatworthy, Buick, Hankins, Weinman y Horne, 2005).

En el caso que aquí se presenta, se utilizará el método de clúster K-means y su cálculo se realizará mediante el programa SPSS V.24 con licencia de la Universitat de València. Ante la necesidad de indicar a priori el número de clústeres a extraer y dado el carácter exploratorio del estudio, se ha desarrollado un procedimiento de segmentación (Alcantud, 1985; Aldenderfer, 1985). Partiendo de una primera partición en dos grupos, se ha seguido segmentando cada uno de ellos sucesivamente hasta que el número de individuos que forman un grupo no permita seguir la segmentación o las diferencias entre las variables utilizadas pierdan significación mediante el ANOVA de contraste final. Una vez alcanzado este objetivo, sobre la clasificación final, se realizará 
VALIDEZ DIAGNÓSTICA DE LA ESCALA DE DESARROLLO MERRILL PALMER-R UTILIZADA EN LA

EVALUACIÓN DE ACCESO A LOS CENTROS DE DESARROLLO INFANTIL Y ATENCIÓN TEMPRANA FRANCISCO ALCANTUD-MARÍN, JAVIER PÉREZ-BOU Y YURENA ALONSO-ESTEBAN

un Análisis Discriminante de forma complementaria con la finalidad de estudiar la consistencia de los grupos formados (Alcantud, 1985).

\section{Resultados}

Se realizaron diferentes análisis de clúster K-means, solicitando la segmentación del grupo de dos en dos, obteniendo los resultados que se expresan en el dendograma de la Figura 2. La verificación de clústeres potenciales será necesaria y debe incluir procedimientos de validación externa, como la comparación de clústeres sobre datos no incluidos en el análisis (Aldenderfer, 1985).

\section{FIGURA 2. Relación de las sucesivas agrupaciones desarrolladas por segmentación}

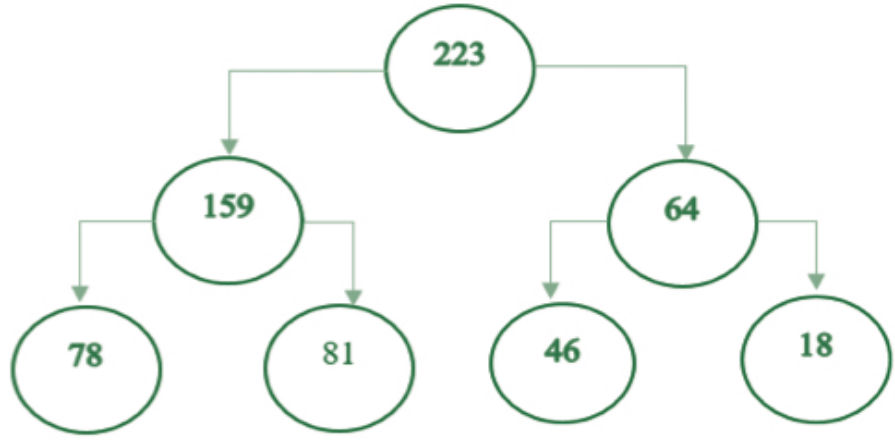

Con la finalidad de contrastar los resultados de la clasificación con el criterio externo del diagnóstico de cada caso, hemos confeccionado la Tabla 5, en ella se observa que el grupo 1.1 está formado por la mayoría $(68,75 \%)$ de los casos que alcanzaron la normalidad en el momento del alta en el centro. El clúster 1.2 está formado por la mayoría de los niños/as $(47,76 \%)$ que en el momento del alta fueron

\begin{tabular}{|l|r|r|r|r|r|}
\hline \multirow{2}{*}{ Tabla 5. Distribución del número de casos según pertenencia a cada clúster y diagnóstico } \\
\hline & \multicolumn{5}{|c|}{ Clúster } \\
\cline { 2 - 7 } & 1.1 & 1.2 & $\mathbf{2 . 1}$ & $\mathbf{2 . 2}$ & Total \\
\hline Normalidad (sin diagnóstico) & 44 & 18 & 2 & 0 & 64 \\
\hline Trastorno Global del Desarrollo & 11 & 32 & 15 & 9 & 67 \\
\hline Discapacidad Intelectual & 2 & 2 & 3 & 3 & 10 \\
\hline Trastorno del Espectro Autista & 3 & 9 & 19 & 5 & 36 \\
\hline Trastorno Motor & 4 & 5 & 1 & 0 & 10 \\
\hline Trastorno del Lenguaje & 14 & 15 & 6 & 1 & 36 \\
\hline Total & 78 & 81 & 46 & 18 & 223 \\
\hline
\end{tabular}

(C) Ediciones Universidad de Salamanca / CC BY-NC-ND

Siglo Cero, vol. 50 (1), n. ${ }^{\circ}$ 269, 2019, enero-marzo, pp. 53-73

$$
-64-
$$


VALIDEZ DIAGNÓSTICA DE LA ESCALA DE DESARROLLO MERRILL PALMER-R UTILIZADA EN LA

EVALUACIÓN DE ACCESO A LOS CENTROS DE DESARROLLO INFANTIL Y ATENCIÓN TEMPRANA FRANCISCO ALCANTUD-MARÍN, JAVIER PÉREZ-BOU Y YURENA ALONSO-ESTEBAN

etiquetados como Trastorno Global del Desarrollo. El clúster 2.1 se forma con la mayoría $(52,78 \%)$ de los niños/s diagnosticados como TEA. El clúster 2.2 se forma por una miscelánea de todos los diagnósticos con la característica de tener los valores en la MPR más bajos, por lo que se puede inferir también que se trata de niños/ as con un trastorno más grave. Este último clúster es además el que menos número de casos aglutina.

En la Tabla 6 aparecen las medias de las variables utilizadas para definir los cuatro clústeres. Tal como se puede observar, existe un cierto orden de gravedad expresado por la proximidad a la normalidad del clúster $1.1(\overline{\mathrm{x}}=100)$ y el más grave con puntuaciones muy bajas correspondiente al clúster 2.2.

\begin{tabular}{|c|c|c|c|c|c|c|c|c|}
\hline & \multicolumn{8}{|c|}{ Clúster } \\
\hline & \multicolumn{2}{|c|}{1.1} & \multicolumn{2}{|c|}{1.2} & \multicolumn{2}{|c|}{2.1} & \multicolumn{2}{|c|}{2.2} \\
\hline & $\bar{x}$ & $\sigma$ & $\bar{x}$ & $\sigma$ & $\overline{\mathrm{x}}$ & $\sigma$ & $\bar{x}$ & $\sigma$ \\
\hline Cognición & 102,23 & 10,20 & 85,26 & 7,94 & 65,69 & 9,34 & 39,06 & 10,48 \\
\hline Motricidad fina & 100,81 & 10,51 & 85,71 & 9,81 & 65,07 & 11,01 & 32,44 & 12,02 \\
\hline Motricidad gruesa & 96,56 & 15,86 & 86,73 & 15,07 & 81,32 & 17,63 & 58,25 & 27,44 \\
\hline Coordinación visomotora & 99,71 & 9,51 & 82,93 & 10,22 & 60,57 & 15,26 & 23,50 & 14,07 \\
\hline Lenguaje receptivo & 98,61 & 12,45 & 84,67 & 9,06 & 64,27 & 12,69 & 34,94 & 17,37 \\
\hline Lenguaje expresivo & 85,26 & 16,98 & 69,08 & 13,16 & 54,54 & 19,27 & 32,72 & 17,84 \\
\hline Socioemocional & 93,11 & 10,56 & 83,11 & 9,01 & 70,98 & 12,40 & 64,72 & 18,58 \\
\hline Conducta autocuidado y adaptativa & 99,76 & 11,74 & 87,55 & 11,58 & 78,24 & 17,28 & 65,06 & 12,94 \\
\hline $\mathrm{N}$ & \multicolumn{2}{|c|}{78} & \multicolumn{2}{|c|}{81} & \multicolumn{2}{|c|}{46} & \multicolumn{2}{|c|}{18} \\
\hline
\end{tabular}

Como puede verse en la Figura 3, al comparar los perfiles de los cuatro grupos se observa una forma semejante con coincidencias y discrepancias más allá de la magnitud de sus puntuaciones. Así, por ejemplo, los cuatro grupos manifiestan un descenso en las puntuaciones de la subprueba de "Lenguaje expresivo" como factor común. En cuanto a la motricidad, el comportamiento parece diferencial, así la prueba de "motricidad fina" y la de "coordinación visomotora" bajan en los clústeres 2.1 y 2.2. mientras que la de "motricidad gruesa" se mantiene más uniforme, al igual que el resto de las subpruebas.

Con la finalidad de estudiar la capacidad discriminante de cada una de las variables o componentes de la MP-R, se realizó un análisis discriminante paso a paso. En la Tabla 7, se presenta el orden de participación en la creación de las funciones discriminantes. Obsérvese que la subprueba que mejor discrimina los cuatro grupos es la de "Cognición", seguida de "Coordinación Visomotora", "Desarrollo Socioemocional", "Lenguaje expresivo" y por último la subprueba de "Motricidad Gruesa". El resto de subpruebas no resultan discriminantes entre los cuatro grupos o clústeres obtenidos con anterioridad. 
VALIDEZ DIAGNÓSTICA DE LA ESCALA DE DESARROLLO MERRILL PALMER-R UTILIZADA EN LA

EVALUACIÓN DE ACCESO A LOS CENTROS DE DESARROLLO INFANTIL Y ATENCIÓN TEMPRANA FRANCISCO ALCANTUD-MARÍN, JAVIER PÉREZ-BOU Y YURENA ALONSO-ESTEBAN

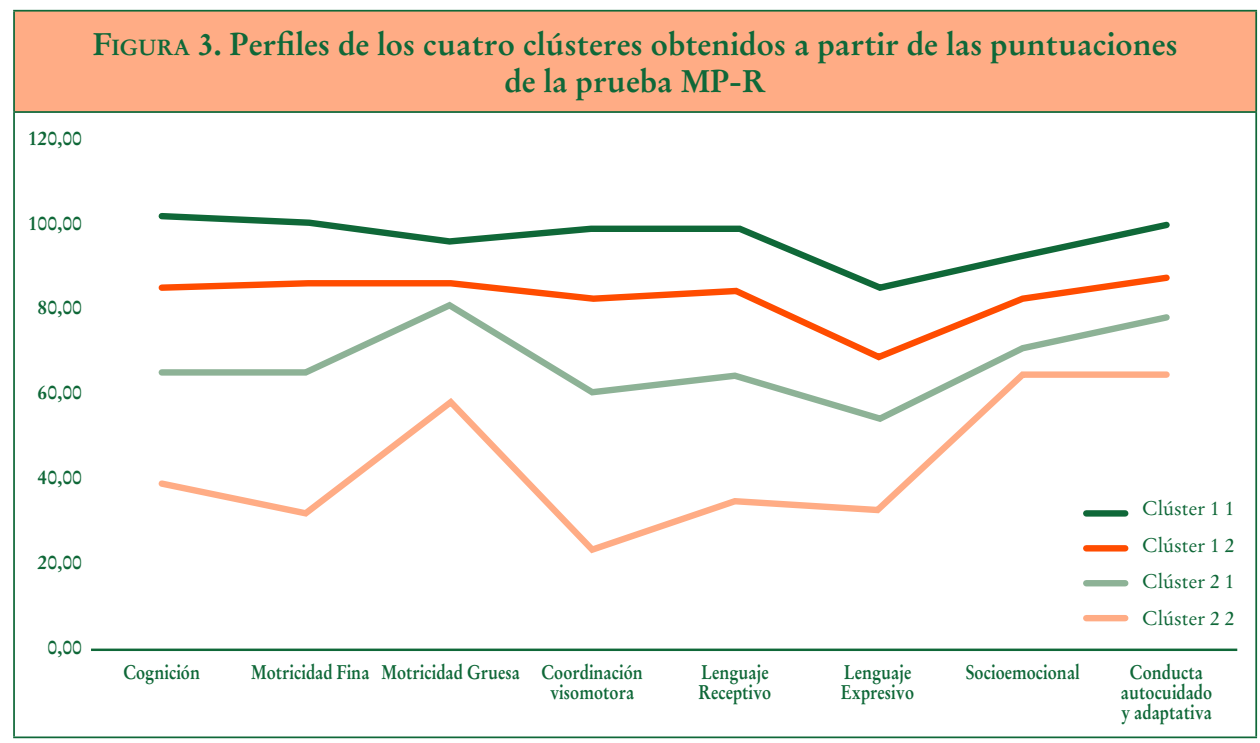

\begin{tabular}{|l|l|r|r|r|}
\hline \multicolumn{5}{|c|}{$\begin{array}{c}\text { Tabla 7. Resultados de selección de variables por el procedimiento de análisis } \\
\text { discriminante paso a paso }\end{array}$} \\
\hline \multirow{2}{*}{$\begin{array}{l}\text { Pasos } \\
1\end{array}$} & Tolerancia & $\begin{array}{c}\text { F } \\
\text { Para eliminar }\end{array}$ & $\begin{array}{c}\text { Lambda } \\
\text { Wilks }\end{array}$ \\
\hline \multirow{4}{*}{2} & Cognición & 1,000 & 300,822 & \\
\cline { 2 - 5 } & Cognición &, 830 & 31,775 &, 216 \\
\hline \multirow{4}{*}{3} & Cognición &, 830 & 21,716 &, 195 \\
\cline { 2 - 5 } & Coordinación visomotora &, 829 & 23,762 &, 170 \\
\cline { 2 - 5 } & Socioemocial &, 822 & 22,230 &, 167 \\
\hline \multirow{4}{*}{4} & Cognición &, 990 & 12,879 &, 150 \\
\cline { 2 - 5 } & Coordinación visomotora &, 806 & 15,371 &, 140 \\
\cline { 2 - 5 } & Socioemocial &, 792 & 25,475 &, 156 \\
\cline { 2 - 5 } & Lenguaje receptivo &, 950 & 8,085 &, 128 \\
\hline \multirow{4}{*}{5} & Cognición &, 911 & 7,649 &, 128 \\
\cline { 2 - 5 } & Coordinación visomotora &, 803 & 11,985 &, 118 \\
\cline { 2 - 5 } & Socioemocial &, 788 & 24,685 &, 136 \\
\cline { 2 - 5 } & Lenguaje receptivo &, 938 & 8,967 &, 114 \\
\cline { 2 - 5 } & Motricidad gruesa &, 851 & 11,306 &, 117 \\
\hline
\end{tabular}

En la Tabla 8 se presentan los coeficientes de correlación de cada variable con las tres funciones discriminantes y los valores propios y el porcentaje de varianza explicado por cada función. 
VALIDEZ DIAGNÓSTICA DE LA ESCALA DE DESARROLLO MERRILL PALMER-R UTILIZADA EN LA

EVALUACIÓN DE ACCESO A LOS CENTROS DE DESARROLLO INFANTIL Y ATENCIÓN TEMPRANA FRANCISCO ALCANTUD-MARÍN, JAVIER PÉREZ-BOU Y YURENA ALONSO-ESTEBAN

\section{Tabla 8. Coeficientes de las funciones discriminantes en las variables seleccionadas}

\begin{tabular}{|l|r|r|r|}
\hline \multirow{2}{*}{ Cognición } & \multicolumn{3}{|c|}{ Funciones } \\
\cline { 2 - 4 } & \multicolumn{1}{|c|}{1} & \multicolumn{1}{c|}{2} & \multicolumn{1}{c|}{3} \\
\hline Visomotora & $0,71 *$ & $-0,21$ & $-0,05$ \\
\hline Motricidad Fina & $0,66^{*}$ & 0,42 & $-0,61$ \\
\hline Lenguaje Receptivo & $0,55^{*}$ & 0,19 & $-0,28$ \\
\hline Socioemocional & $0,42 *$ & $-0,24$ & $-0,07$ \\
\hline Conducta Adaptativa & 0,29 & $-0,76^{*}$ & $-0,04$ \\
\hline Motricidad Gruesa & 0,09 & $-0,22 *$ & 0,07 \\
\hline Lenguaje Expresivo & 0,21 & 0,43 & $0,56 \%$ \\
\hline Valores propios & 0,34 & $-0,17$ & $0,45^{*}$ \\
\hline$\%$ Varianza & 8,15 & 0,06 & 0,01 \\
\hline
\end{tabular}

* Mayor correlación absoluta entre cada variable y cada función discriminante.

Parece claro que la función discriminante 1 está definida por las subpruebas de “cognición”, “coordinación visomotora”, "motricidad fina” y "lenguaje receptivo", determina un continuo de gravedad en el trastorno del desarrollo, explicando un $99,1 \%$, mientras que la función discriminante 2 se define por las subpruebas "socioemocional” y "conducta adaptativa” y en el polo opuesto las subpruebas de "coordinación visomotora” y "motricidad gruesa”. Por último, la tercera función discriminante se define por las subpruebas de" lenguaje expresivo" y "motricidad gruesa” en oposición también a la subprueba de "coordinación visomotora”. Al representar gráficamente las dos primeras funciones (Figura 4), se observa que la primera (horizontal) clasifica a los niños/as desde el clúster 1.1 (considerado como el menos grave o más próximo a la normalidad) hasta el clúster 2.2 (considerado como el más grave desde el punto de vista del desarrollo cognitivo). Las subpruebas que diferenciarían unos de otros son las que definen la función. La segunda dimensión representada tiene menos variabilidad, no obstante, aparecen niños/as del clúster 2.1 con puntuaciones negativas más altas (recuérdese que en este clúster se agrupaban el mayor porcentaje de niños diagnosticados con TEA y esta función se caracteriza por puntuaciones negativas de las subpruebas de "desarrollo socioemocional" y "conducta adaptativa”).

Es importante observar como los niños/as del clúster 1.1, 1.2 y 2.1 están más agrupados (menores dispersiones). Mientras que en el clúster 2.2 aparecen los casos más dispersos, aunque el número de casos sea inferior. Se podría decir que conforme se incrementa la gravedad también los grupos son más heterogéneos.

En función de los valores de las funciones discriminantes, se pueden predecir los grupos de pertenencia. Si analizamos la capacidad predictiva se observa que el $93,7 \%$ de casos (Tabla 9) están correctamente clasificados. Se puede considerar que la clasificación obtenida es óptima. 
FIGURA 4. Representación gráfica del mapa territorial de los cuatro grupos

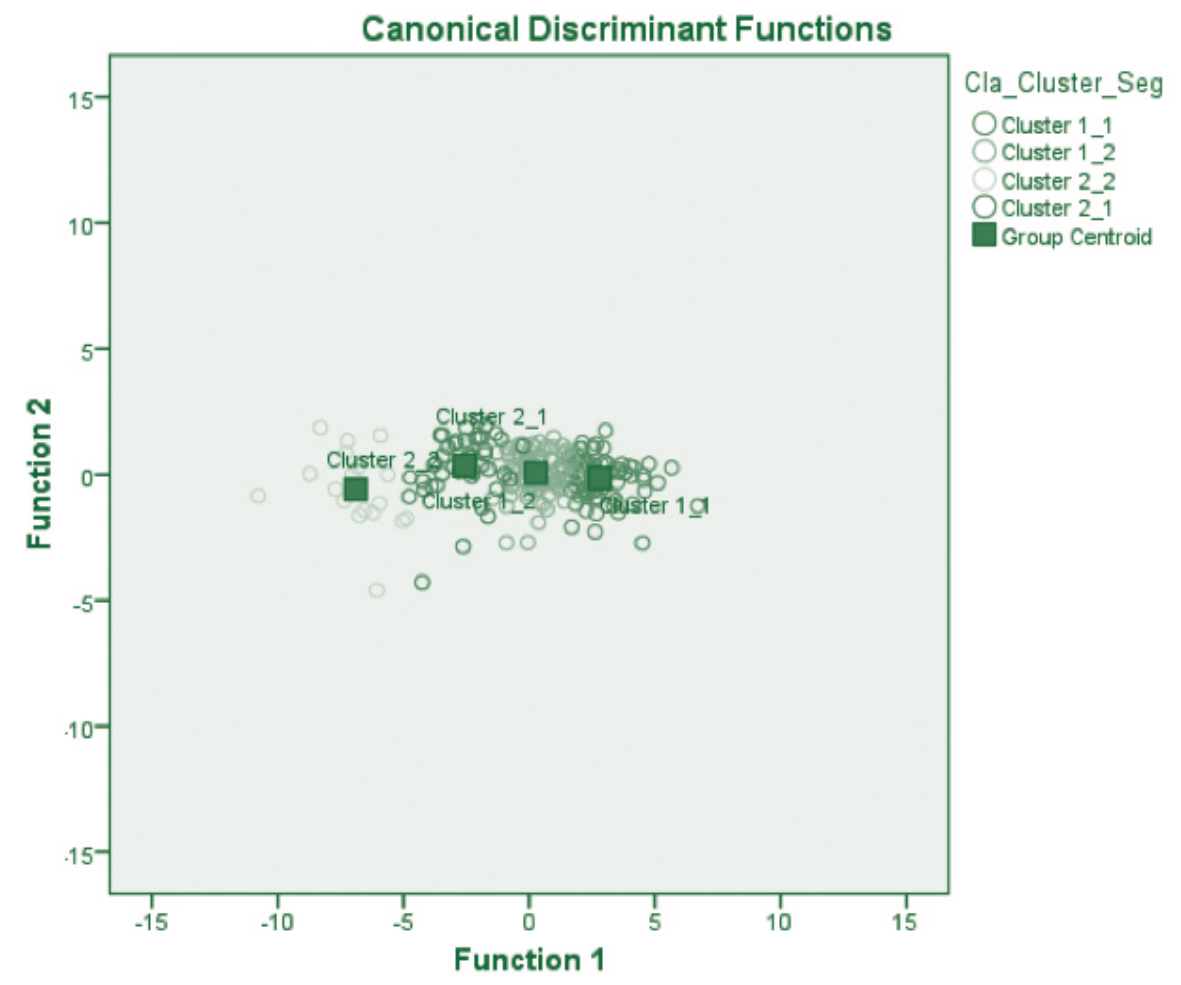

\begin{tabular}{|c|c|c|c|c|c|c|}
\hline \multirow{2}{*}{\multicolumn{2}{|c|}{1}} & \multicolumn{4}{|c|}{ Clasificación predicha } & \multirow{2}{*}{ Total } \\
\hline & & 2 & 3 & 4 & & \\
\hline \multirow{4}{*}{$\begin{array}{l}\text { Clasificación } \\
\text { Original }\end{array}$} & Clúster 1.1 & $74(94,9 \%)$ & 4 & 0 & 0 & 78 \\
\hline & Clúster 1.2 & 3 & $77(95,1 \%)$ & 0 & 1 & 81 \\
\hline & Clúster 2.1 & 0 & 5 & $40(87,0 \%)$ & 1 & 46 \\
\hline & Clúster 2.2 & 0 & 0 & 0 & $18(100 \%)$ & 18 \\
\hline
\end{tabular}

a. El 93,7\% de los casos originales estaban bien clasificados.

\section{Discusión y conclusiones}

En general en los CDIAT, dado el carácter ambulatorio de su intervención, se pueden definir diferentes modalidades de intervención según la intensidad, recursos o 
VALIDEZ DIAGNÓSTICA DE LA ESCALA DE DESARROLLO MERRILL PALMER-R UTILIZADA EN LA

EVALUACIÓN DE ACCESO A LOS CENTROS DE DESARROLLO INFANTIL Y ATENCIÓN TEMPRANA FRANCISCO ALCANTUD-MARÍN, JAVIER PÉREZ-BOU Y YURENA ALONSO-ESTEBAN

posicionamiento que se utilicen. La administración de una evaluación utilizando una escala como la aquí analizada no es algo sencillo, sobre todo si esta forma parte de la evaluación inicial en el momento de alta en el CDIAT. Se hará necesario que la persona que va a realizar la evaluación utilice varias sesiones de trabajo solo para conocer al niño/a y que este se sienta cómodo. En estas sesiones se hace indispensable la presencia de los progenitores o responsables del cuidado del niño. También es un momento adecuado para que, mediante juegos programados, observemos la conducta y habilidades del niño/a. Una vez que el profesional ha conseguido establecer un "rapport" adecuado, es cuando se pueden iniciar las sesiones de evaluación propiamente dichas. Previamente a la evaluación, durante la primera entrevista a la familia recabaremos información complementaria que nos permitirá conocer mejor al niño/a. También es de gran ayuda la aportación de videos domésticos de eventos familiares donde haya participado el niño y, si es posible, la observación del niño en contextos naturales (en su domicilio o en la escuela infantil donde asista si es el caso). El uso de herramientas de evaluación como la Escala Merrill Palmer-R en su momento inicial permite realizar una valoración del caso. El objetivo de este artículo era conocer el valor predictivo de esta escala respecto al diagnóstico del niño/a y su nivel de gravedad con la finalidad de poder utilizarla como para determinar los recursos del CDIAT asignados al caso.

Aunque con las limitaciones derivadas de que los datos se originan en un único centro, los resultados demuestran que las puntuaciones en la escala MP-R obtenidas en el momento del alta en el centro permiten un pronóstico en términos estadísticos más o menos ajustado a los diagnósticos que alcanzaron los niños en el momento de la baja en el centro. Resulta obvio que no es suficiente para un pronóstico exacto contar solo las puntuaciones de una escala. Se deberá complementar esta información con la información médica, familiar, la obtenida por la observación del niño, etc. Debe tenerse en cuenta, no obstante, que la evaluación previa es el primer eslabón de todo el proceso de intervención y, por tanto, a lo largo del tiempo los datos obtenidos en esa evaluación previa variarán y su pronóstico también.

Los resultados que se muestran en este estudio definen que los niños/as atendidos en el CDIAT analizado tienen diversos niveles de gravedad al margen del diagnóstico. Es más, tal como marca el DSM-5 (APA, 2013), se debe acompañar a la etiqueta diagnóstica de una valoración de la gravedad. Quizás con este dato la clasificación obtenida hubiera sido más nítida. No obstante, en el caso de los niños/as atendidos en un CDIAT, la valoración de la gravedad puede resultar compleja por la edad del niño. En el momento de baja en el centro, un porcentaje alto de los casos no tienen un diagnóstico concreto debido a que por la edad no se ha podido aplicar un instrumento formal de evaluación de la inteligencia. Esto lleva a que la categoría definida como “Trastornos Globales del Desarrollo” se convierta en un cajón de sastre que irá paulatinamente definiéndose.

En contraposición, las familias (los padres/madres principalmente) se encuentran sometidas a un alto nivel de estrés ante la situación de incertidumbre producida por la falta de información sobre el posible diagnóstico. La aplicación de una escala de desarrollo como la aquí analizada puede permitir, de forma objetiva, aportar luz sobre el nivel de gravedad y la necesidad de apoyos futuros y/o tomar decisiones hacia qué 
tipo o intensidad de intervención desarrollar. Es obvio que la aplicación de una escala de desarrollo solo es el inicio de un camino que deberá complementarse con otras pruebas y cotejarla con información recogida por la observación diaria en el comportamiento del niño/a. Ahora bien, en el caso de que las puntuaciones en esta escala sean no significativas, dispondremos de una valoración objetiva en la que se sustente la decisión de baja en el centro.

Así, por ejemplo, el grupo formado por los niños/as y niñas del clúster 1.1 tiene un perfil en todas las subpruebas dentro de la normalidad $(\geq \bar{x} \pm 1 \sigma)$. En consecuencia, si no se dispone de más información, son candidatos a una intervención de seguimiento o incluso de baja en el centro. En el caso del resto de niños/as se puede iniciar la intervención programando una intervención que intente cubrir los déficits en las áreas evaluadas y/o proseguir realizando evaluaciones más específicas. La evaluación objetiva mediante el uso de instrumentos como la MP-R no descarta el uso de otras herramientas objetivas más especializadas en áreas concretas del desarrollo o más cualitativas como los registros observacionales. De hecho, la propia escala MP-R incluye subpruebas cuyas puntuaciones dependen de la respuesta de los padres/ madres (Socioemocional, Conducta adaptativa o Lenguaje Infantil, entre otras). Es evidente que la conducta del niño/a no será la misma en el centro durante la evaluación que en su entorno natural, por tanto, será necesario también contrastar la información obtenida con la observación en el medio natural.

El disponer en un sistema de gestión del centro del mecanismo para, una vez introducidas las puntuaciones de la escala de desarrollo, que nos asigne un perfil probable de intervención y, con ello, seleccione de una base de datos los objetivos y actividades asociadas al perfil facilita la labor de los profesionales. No se trata de generar automáticamente un programa de intervención solo en base a una prueba. Se trata de ayudar a seleccionar, de entre los centenares de objetivos y actividades del currículum de desarrollo infantil, aquellos que más probablemente se deberían seleccionar para confeccionar el programa individual. Será el profesional, de forma consensuada con la familia, el que deberá aceptar o modificar, eliminar o incluir objetivos en un proceso que siempre será menos laborioso que si debemos confeccionarlo sin ayuda y orientación. Este sistema ha sido incorporado con éxito en el SIGAT 2.04 (Pérez, 2015). Este sistema nos facilita un listado de objetivos y actividades para confeccionar los programas y después registrar los resultados de forma que, con el tiempo, podamos tener evidencias de los mismos.

El modelo de intervención transdisciplinar nos obliga a tener una comunicación constante con otros profesionales con diferentes perfiles, tanto en el mismo centro como fuera de él. En nuestra opinión y para mejorar la objetividad de nuestra información se hace necesario el uso de instrumentos de evaluación objetiva que permitan tanto determinar el nivel del desarrollo del niño/a en un momento dado como su seguimiento en evaluaciones posteriores. Además, con la finalidad de optimizar el uso de los escasos recursos que en la actualidad poseen los CDIAT, disponer de una herramienta que permita valorar la gravedad de los casos atendidos con la finalidad de administrar la lista de espera de forma coherente.

4 SISGAT 2.0: Sistema de Gestión en Atención Temprana versión 2.0. 
VALIDEZ DIAGNÓSTICA DE LA ESCALA DE DESARROLLO MERRILL PALMER-R UTILIZADA EN LA

EVALUACIÓN DE ACCESO A LOS CENTROS DE DESARROLLO INFANTIL Y ATENCIÓN TEMPRANA FRANCISCO ALCANTUD-MARÍN, JAVIER PÉREZ-BOU Y YURENA ALONSO-ESTEBAN

Cualquier escala de desarrollo nos puede informar del estado del niño/a, en el caso que nos ocupa, los resultados obtenidos con la aplicación de la escala MP-R nos demuestran que puede ser de gran ayuda tanto a la hora de determinar los recursos necesarios para la atención como a la hora de establecer hipótesis diagnósticas que confirmaremos posteriormente con pruebas complementarias o planificar los contenidos de los programas de intervención.

En la medida que nuestra comunidad disponga de recursos de atención temprana especializados, el uso de este tipo de herramientas permitiría, de forma objetiva, desarrollar la derivación hacia ellos. En nuestra opinión, y siguiendo el modelo sanitario, los centros actuales de atención temprana hacen la función de los centros de salud de atención primaria. Se realizan evaluaciones, algunos descartes, se inician tratamientos más o menos generales, pero para determinados trastornos se requieren centros especializados como puede ser el caso de los Trastornos del Espectro Autista, Parálisis Cerebral Infantil o trastornos sensoriales como la ceguera.

Como línea de actuación futura, se hace necesario el desarrollo de protocolos de actuación según el diagnóstico y su gravedad que orienten a profesionales y familiares sobre cuál o cuáles pueden ser los métodos y contenidos de intervención más adecuados en función de las evidencias encontradas.

\section{Referencias bibliográficas}

Alcantud, F. (1985). Técnicas de clasificación. Valencia: Ediciones Universitarias de Valencia.

Alcantud, F. y Alonso, Y. (2016). Predictive value of the Merrill-Palmer R Scale applied during the first year of live. En Colegio Oficial de Psicólogos de Madrid (Ed.), Psicología Educativa. doi: http://dx.doi.org/10.1016/j.pse.2016.01.001.

Aldenderfer, M. (1985). Cluster Analysis. London: SAGE Publications Inc.

APA (2013). Diagnostic and statistical manual of mental disorders (DSM-5). American Psychiatric Pub.

BARNETT, S. (1995). Long term effects of early childhood programs on cognitive and school outcomes. The Future of Children, 5 (3), 25-50. doi: 10.2307/1602366.

Bayley, N. (2006). Bayley scales of infant and toddler development. 3rd Edn Screening test Manual. San Antonio Texas: Pearson Education Inc.

Belinchon, M. y Olivar, J. (2003). Trastornos del espectro autista en personas con alto nivel de funcionamiento: Diferenciación funcional mediante análisis multivariado. Acción Psicológica, 2 (3), 223-238. Recuperado el 10 de octubre de 2015, de http://revistas.uned.es/ index.php/accionpsicologica/article/view/533/471.

Casto, G. y Mastropieri, M. (1986). The Efficacy of Early Intervention Programs: A MetaAnalysis. Exceptional Children, 52 (5), 417-424.

CESM-CV Sindicato Médico de la Comunidad Valenciana (2009). La Situación Actual de los Pediatras y la Pediatría en la Comunidad Valenciana. Valencia: Sindicato Médico de la Comunidad Valenciana. Recuperado el 20 de agosto de 2016, de http://cesm-cv.org/wp-content/uploads/2011/03/Situaci\%C3\%B3n-actual-de-la-Pediatr\%C3\%ADa-en-la-CV2.pdf.

Clatworthy, J., Buick, D., Hankins, M., Weinman, J. y Horne, R. (2005). The use and reporting of cluster analysis in health psychology: a review. British Journal of Health Psycho$\log y, 10$ (3), 329-358. doi: 10.1348/135910705X25697. 
VALIDEZ DIAGNÓSTICA DE LA ESCALA DE DESARROLLO MERRILL PALMER-R UTILIZADA EN LA

EVALUACIÓN DE ACCESO A LOS CENTROS DE DESARROLLO INFANTIL Y ATENCIÓN TEMPRANA FRANCISCO ALCANTUD-MARÍN, JAVIER PÉREZ-BOU Y YURENA ALONSO-ESTEBAN

Crespo-Eguílaz, N. y Narbona, J. (2006). Subtipos de trastorno específico del desarrollo del lenguaje: Perfiles clínicos en una muestra de hispanohablantes. Revista de Neurología, S193-S200. Recuperado el 12 de octubre de 2015, de http://www.neurologia.com/pdf/ web/43s01/ws01s193.pdf.

Crespo-Eguílaz, N. y Narbona, J. (2009). Trastorno de aprendizaje procedimental: Características neuropsicológicas. Revista de Neurología, 49, 409-416. Obtenido de http://www. neurologia.com/pdf/web/4908/bc080409.pdf.

Escudero, L. (1977). Reconocimiento de patrones. Madrid: Paraninfo.

GAT (2000). Libro Blanco de Atención Temprana. Madrid: Real Patronato sobre Atención Temprana.

GAT (2004). Organización Diagnóstica de Atención Temprana (ODAT): Manual de uso. Madrid: Real Patronato sobre Discapacidad (Ministerio de Sanidad, Igualdad y Bienestar Social).

GAT (2011). La Realidad Actual de la Atención Temprana en España. Madrid: Real Patronato sobre Discapacidad. Ministerio de Sanidad, Política Social e Igualdad.

Guralnick, M. (1989). Recents developments in Early Intervention Efficacy Research: Implicantions for family involvement in P.L. 99-457. Topics in Early Childhood Special Education, 9 (3), 1-17. doi: 10.1177/027112148900900302.

Guralnick, M. (1997). Second generation research in the field of early intervention. En M. Guralnick (Ed.), The Effectiveness of early intervention (pp. 3.22). Baltimore: Brookes.

Guralnick, M. (1998). The effectiveness of early intervention for vulnerable children: A developmental perspective. American Journal on Mental Retardation, 102, 319-345.

GuRALNICK, M. (2000). Interdisciplinary clinical assessment for young children with developmental disabilities. Baltimore: Brookes.

Guralnick, M. (2017). Early intervention for children with intellectual disabilities: An update. Journal of Applied Research in Intellectual Disabilities, 30, 211-216. doi: 10.1111/jar.12233.

Guralnick, M. (2017). Early intervention for young children with developmental delays: Contribution of the developmental systems approach. En H. Sukkar, C. Dunst y J. KIRKBY, Early childhood intervention: working with families of young children (pp. 17-35). Oxon UK: Routledge.

Guralnick, M. J. y BennetT, F. C. (1987). The effectiveness of early intervention for at-risk and handicapped children. New York: Academic Express.

Josse, D. (1997). Brunet-Lézine Révisé: Échelle de développement psychomoteur de la petite enfance. París: Établissements d'Applications Psychotechniques.

King, G., Strachan, D., Tucker, M., Duwyn, M., Desserud, B. y Shillington, B. (2009). The application of a transdisciplinary model for Early Intervention services. Infants and Young Children, 22 (3), 211-223. doi: 10.1097/IYC.0b013e3181abe1c3.

McCormick, M., Brooks-Gum, J., Buka, S., Goldman, J. y Yu, J. (2006). Early intervention in Low Birth Weight Premature Infants: Results at 18 years of age for the Infant Health and Development Program. Pediatrics, 117, 771-780.

Newborg, J. (2005). Battelle Developmental Inventory, 2nd Edition: User's Guide. Itasca, Illinois: Riverside Publishing.

Pérez, J. (2015). Sistema de ayuda a la toma de decisiones en la valoración de perfiles, a partir del desarrollo de un nuevo modelo de datos en el "Centre Universitari de Diagnòstic $i$ Atenció Primerenca” (CUDAP). Valencia: Universitat de Valencia. Recuperado el 12 de septiembre de 2016, de http://roderic.uv.es/bitstream/handle/10550/42900/Tesis\%20Javier $\% 20 \mathrm{P} \% \mathrm{C} 3 \%$ A 9rez.pdf? sequence=1\&isAllowed=y. 
VALIDEZ DIAGNÓSTICA DE LA ESCALA DE DESARROLLO MERRILL PALMER-R UTILIZADA EN LA

EVALUACIÓN DE ACCESO A LOS CENTROS DE DESARROLLO INFANTIL Y ATENCIÓN TEMPRANA

FRANCISCO ALCANTUD-MARÍN, JAVIER PÉREZ-BOU Y YURENA ALONSO-ESTEBAN

Peters, M. (2013). Determining the Clinical Utility of the Merrill-Palmer-Revised Scales of Developmental in a sample of children with autistic disorder. Doctor of Psychology (PsyD) Paper 110: George Fox University. Obtenido de http://digitalcommons.georgefox.edu/ psyd/110.

Reynolds, A., Temple, J., Robertson, D. y Mann, E. (2001). Long term effects of an early childhood intervention on educational achievement and juvenile arrest. Journal of the American Medical Association, 2339-2346.

RoID, G. (2003). Stanford-Binet Intelligence Scales (5 ${ }^{a}$ Edition). Itasca, Illinois: Riverside Publishing Co.

Roid, G. y Miller, L. (1997). Leiter International Performance Scale-Revised. Wood Dale (Illinois): Stoelting Co.

Roid, G. y SAmpers, J. (2004). Merrill-Palmer Revised Scales of Development. Wood Dale (Illinois): Stoelting Co.

Shonkoff, J. P. y Hauser-Cram, P. (1987). Early Intervention for Disabled Infants and Their Families: A Quantitative Analysis. Pediatrics, 80 (5), 650-658.

Stutsman, R. (1931). Merrill-Palmer Scale of Mental Tests. New York: World Book. 


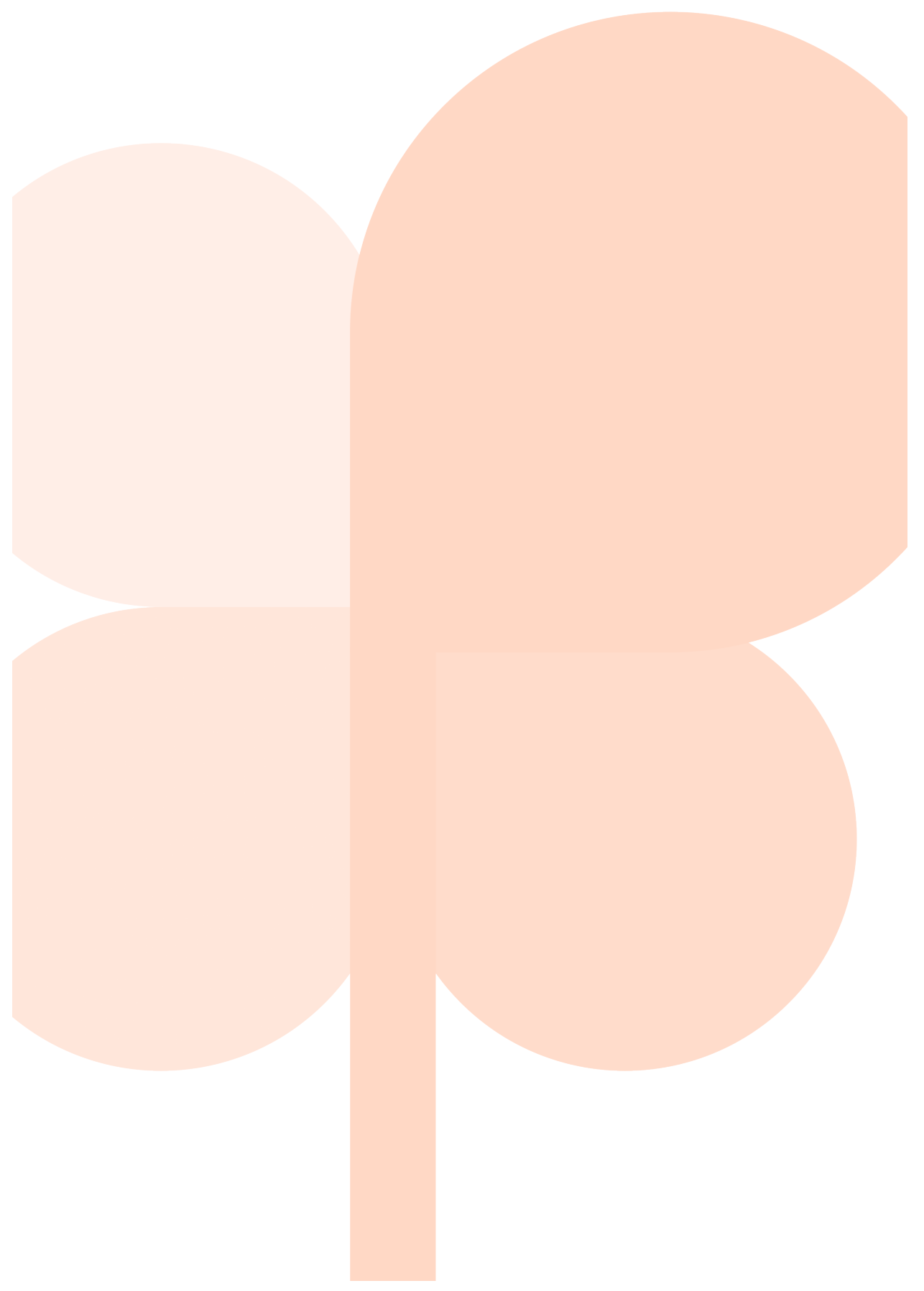

